\title{
Long Term Continuous Collection of High Resolution ECG Signals from a Coronary Care Unit
}

\author{
I Romero Legarreta ${ }^{1}$, MJ Reed ${ }^{3}$, JN Watson ${ }^{2}$, PS Addison ${ }^{1,2}$, N Grubb ${ }^{4}$, \\ GR Clegg ${ }^{3}$, CE Robertson ${ }^{3}$, KAA Fox ${ }^{4}$ \\ ${ }^{1}$ Faculty of Engineering and Computing, Napier University, Edinburgh, UK, \\ ${ }^{2}$ Cardiodigital, Elvingston, UK, ${ }^{3}$ Department of Emergency Medicine, Royal Infirmary of \\ Edinburgh, Edinburgh, UK, ${ }^{4}$ Department of Cardiology, Royal Infirmary of Edinburgh, Edinburgh, \\ UK
}

\begin{abstract}
Cardiovascular disease is the commonest cause of death in Western society. For this reason much effort has been dedicated to the study of its origins, prevention and treatment. Our group has a specific interest in the analysis of the Electrocardiogram (ECG) in life threatening cardiac arrhythmias and its antecedents, with a view to providing diagnostic tools and information to facilitate patient resuscitation. For development and testing of analytical tools we require a relevant library of real ECG signals. In this paper we describe the acquisition and storage of a continuous collection of ECG signals obtained from the Coronary Care Unit $(C C U)$ at the Royal Infirmary of Edinburgh, UK.
\end{abstract}

\section{Introduction}

Cardiovascular disease is the commonest cause of death in Western society. For this reason much effort has been dedicated to the study of its prevention. New techniques based on signal digital processing of ECGs are of specific interest to our group [1].

For the development and testing of these techniques, a realistic library of ECG signals is necessary [2,3]. There are a variety of databases already available for this purpose [4]. In this paper we describe the acquisition and storage of a new 'long-term' or 'continuous' database containing ECG signals obtained from the $\mathrm{CCU}$ at the Royal Infirmary of Edinburgh.

\section{Materials and methods}

Equipment was installed within the CCU at the Royal Infirmary of Edinburgh to collect ECG signals continuously from all six beds within the unit [Figure 1]. Protocols were formulated for data collection and archiving of the collected signals. The data collected was archived over eighteen months (requiring approximately
$3 \mathrm{~GB}$ of storage per week for a signal sample rate of $500 \mathrm{~Hz}$ ). The technology is now available to perform this task in a compact, easily accessible form at a reasonably affordable price. Archiving the whole ECG time series for each patient, including arrhythmic episodes such as ventricular fibrillation (VF) and ventricular tachycardia (VT) [Figure 2], allows subsequent analysis of the signal using a variety of techniques. It will also allow the complete data set to be revisited in the future, when further types of analysis could be performed. The database comprises a large number of patients with very varied cardiac pathologies and will be extremely useful for research into many areas of cardiovascular disease. To date we have employed two time-frequency methods, the Continuous Wavelet Transform (CWT) [Figure 3], and the Short Term Fourier Transform (STFT) to analyse ECG signals immediately before the onset of ventricular arrhythmias.

\section{High Resolution ECG (HRECG)}

The ECG collected has a sample frequency of $500 \mathrm{~Hz}$, which allows a frequency analysis up to $250 \mathrm{~Hz}$ due to the Nyquist theorem. This cut-off frequency is usually enough for the study of the most relevant information contained in the ECG. $\mathrm{P}$ and $\mathrm{T}$ waves have a frequency range up to $20 \mathrm{~Hz}$ and the QRS complexes up to 40 or 50 $\mathrm{Hz}$. Some high frequency waves within the ECG have higher ranges up to 100 or $150 \mathrm{~Hz}$. Our collected signals are relatively clean from power interferences. (These interferences are usually at $50-60 \mathrm{~Hz}$ that is still inside the band of interest in a conventional ECG.) Other databases such as the MIT/BIH database exhibit significant interference of this type [4].

For each sample we used 16 bit resolution, which allowed $2^{16}$ levels of quantization. This is a high enough resolution for the analysis of micro potential waves within the ECG signal. This type of analysis requires advanced techniques of post-processing in order to remove the noise that usually has higher voltage than 
these micro voltage signals. The noise is uncorrelated with the ECG however micro potential waves are correlated with the ECG. This characteristic allows the use of signal averaging methods to remove this noise and study these low voltage signals.

\section{Results}

After a number of months setting up and testing the equipment within the Royal Infirmary of Edinburgh, the data collection project was initiated at the beginning of October 2001. A research nurse collects patient information on a daily basis. ECG data is continuously recorded on a computer in the hospital, from where it is archived on a weekly basis. A number of teething problems were encountered and overcome during the first few months of operation. A working system was finally up and running by December 2001. In the year and a half period since the normal running of the system, more than 20,000 hours of ECG data have been collected. Both Fourier and Wavelet time-frequency tools have been employed in the analysis of the collected waveforms. Although analysis of the data is at an early stage, it is clear from our preliminary results that the wavelet transform offers better all-round resolution due to its inherent flexible time-frequency window. We believe that the continuous wavelet transform will establish itself as an important tool for the digital processing of ECG data. Unlike the STFT, which employs a fixed time frequency window and the complex sinusoid as analysing function, the CWT can offer arbitrarily high resolution at both low and high frequency through its flexible time frequency window, and the choice of analysing wavelet function can offer some limited ability to match the specific signal features. The flexible window has been found particularly useful in detecting previously unseen high frequency structure in the VF waveform [1].

A database programmed in Microsoft Access has been designed to record details of all the patients who develop a ventricular arrhythmia during their stay in CCU. This enables us to quickly and efficiently access both arrhythmic events, and further medical details of the patients involved. The details of the patient's admission, their underlying diagnosis, past medical history, current medication, laboratory investigations on admission, time of adverse events, treatment administrated and the outcome after treatment, were all obtained from hospital records after the patient's discharge. Some screen shots from this database are shown in figures 4 and 5. Each patient who develops recorded arrhythmias whilst in the $\mathrm{CCU}$ unit is enterd on this database. Figure 4 contains a screen shot where background medical details of each patients is inputed. Figure 5 contains a second screen shot where details of each arrhythmic episode are recorded.

\section{Conclusions}

The archiving of a complete set of ECG data from the $\mathrm{CCU}$ over this prolonged time period represents a useful resource within the biomedical engineering community. In addition to our work, which focuses on the pre-VF ECG, the collected data is useful for the retrospective study of a wide variety of ECG signal abnormalities. There has been much recent work concerned with ECG signal features that may predict malignant arrhythmias including VF. We believe that time-frequency-based analysis methods, and in particular the wavelet transform, can be the foundation of powerful new tools for the analysis of ECG signal, as they cope well with transient and local nonstationarities in the signal. In addition, the flexible time-frequency window associated with the wavelet transform allows for arbitrarily high resolution of pertinent signal features.

\section{References}

[1] Addison PS, Watson JN, Clegg GR, Holzer M, Sterz F, Robertson CE. Evaluating arrhythmias in ECG signals using wavelet transforms. IEEE Engineering in Medicine and Biology 2000;19:383-392.

[2] Värri A, Kemp B, Penzel T, Schlögl A. Standards for Biomedical Signal Databases. IEEE Engineering in Medicine and Biology 2001;20:33-37.

[3] Penzel T, Kemp B, Klösch G, Schlögl A, Hasan J, Värri A, Korhonen I. Acquisition of Biomedical Signals Databases. IEEE Engineering in Medicine and Biology 2001;20:25-32.

[4] Moody GB Mark RG. The Impact of the MIT-BIH Arrhythmia database. IEEE Engineering in Medicine and Biology 2001;20:45-50.

Address for correspondence.

Iñaki Romero Legarreta

Faculty of Engineering and Computing

Napier University

10, Colinton Road

Edinburgh EH10 5DT, UK

inaki@napier.ac.uk 


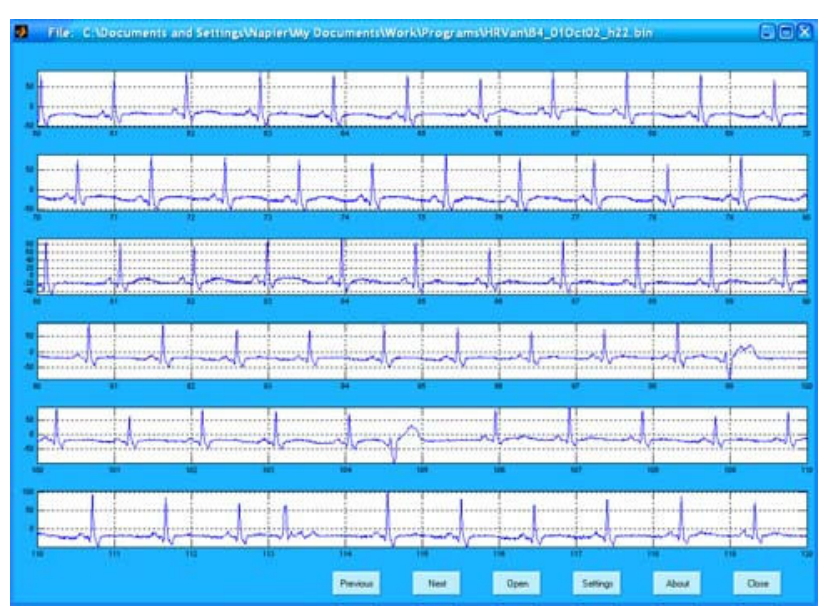

Figure 1. Sample ECG showing both normal and ectopic beats.

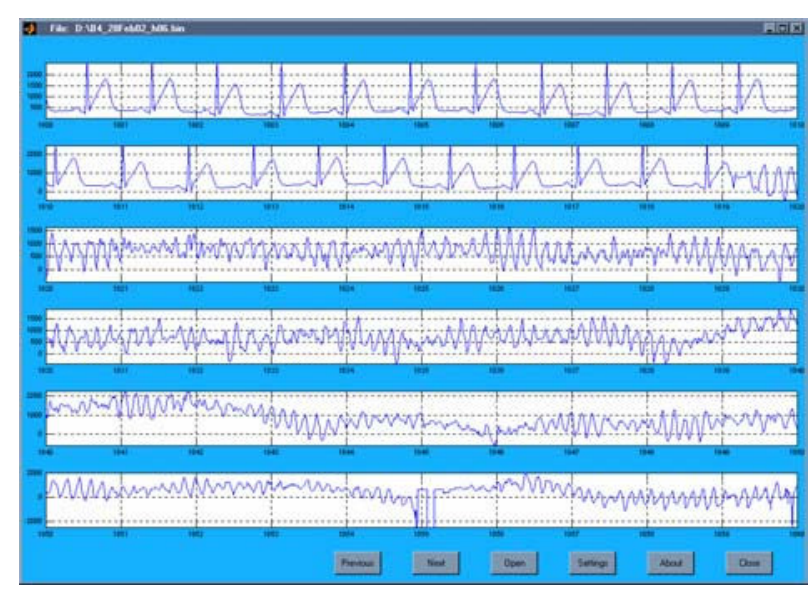

Figure 2. Screen shot from our custom built ECG archiving/display software (developed using Matlab) showing a patient going from sinus rhythm into an episode of VF (All ECG recordings show standard lead II).

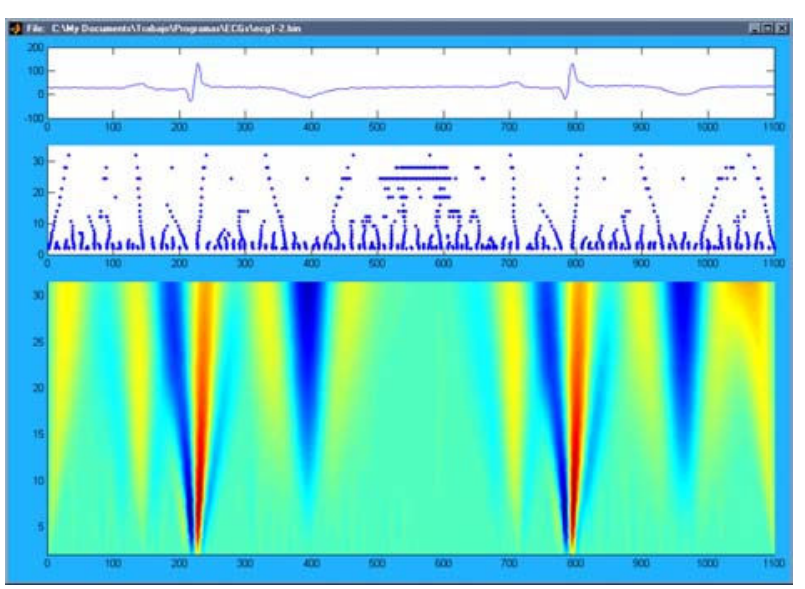

Figure 3. Continuous wavelet transform of a 2 second segment of ECG.

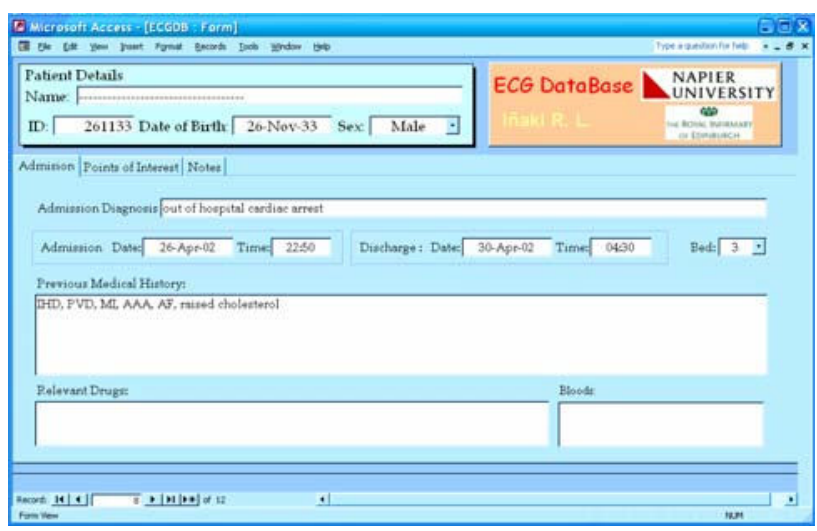

Figure 4. Screen shot from our in house database containing medical details of patients developing recorded arrhythmias whilst in the CCU unit.

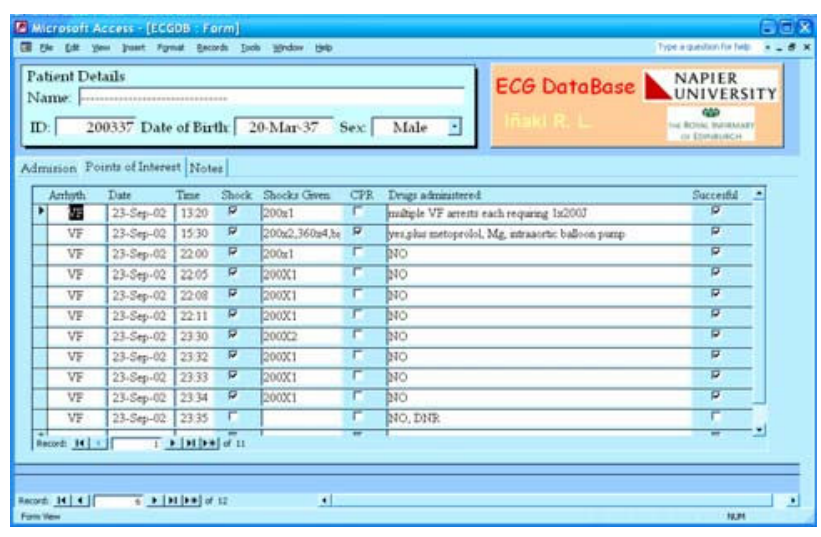

Figure 5. Screen shot from our in house database containing further information of specific arrhythmic events. 